\title{
Traveling Wave Solutions of the Benjamin-Bona-Mahony Water Wave Equations
}

\author{
A. R. Seadawy ${ }^{1,2}$ and A. Sayed ${ }^{2}$ \\ ${ }^{1}$ Mathematics Department, Faculty of Science, Taibah University, Al-Ula 41921-259, Saudi Arabia \\ ${ }^{2}$ Mathematics Department, Faculty of Science, Beni-Suef University, Egypt \\ Correspondence should be addressed to A. R. Seadawy; aly742001@yahoo.com
}

Received 5 August 2014; Revised 1 September 2014; Accepted 4 September 2014; Published 15 October 2014

Academic Editor: Santanu Saha Ray

Copyright (c) 2014 A. R. Seadawy and A. Sayed. This is an open access article distributed under the Creative Commons Attribution License, which permits unrestricted use, distribution, and reproduction in any medium, provided the original work is properly cited.

The modeling of unidirectional propagation of long water waves in dispersive media is presented. The Korteweg-de Vries (KdV) and Benjamin-Bona-Mahony (BBM) equations are derived from water waves models. New traveling solutions of the KdV and BBM equations are obtained by implementing the extended direct algebraic and extended sech-tanh methods. The stability of the obtained traveling solutions is analyzed and discussed.

\section{Introduction}

Many nonlinear evolution equations are playing important role in the analysis of some phenomena and including ion acoustic waves in plasmas, dust acoustic solitary structures in magnetized dusty plasmas, and electromagnetic waves in size-quantized films [1-4]. To obtain the traveling wave solutions to these nonlinear evolution equations, many methods were attempted, such as the inverse scattering method [5], Hirotas bilinear transformation [6], the tanhsech method, extended tanh method, sine-cosine method [7], homogeneous balance method, Bäcklund transformation [8], the theory of Weierstrass elliptic function method [9], the factorization technique $[10,11]$, the Wadati trace method, pseudospectral method, Exp-function method, and the Riccati equation expansion method were used to investigate these types of equations $[12,13]$. The above methods derived many types of solutions from most nonlinear evolution equations [14].

The Benjamin-Bona-Mahony (BBM) equation is well known in physical applications [15]; it describes the model for propagation of long waves which incorporates nonlinear and dissipative effects; it is used in the analysis of the surface waves of long wavelength in liquids, hydromagnetic waves in cold plasma, acoustic-gravity waves in compressible fluids, and acoustic waves in harmonic crystals [15]. Many mathematicians paid their attention to the dynamics of the BBM equation [16].

The BBM equation has been investigated as a regularized version of the $\mathrm{KdV}$ equation for shallow water waves [17]. In certain theoretical investigations the equation is superior as a model for long waves; from the standpoint of existence and stability, the equation offers considerable technical advantages over the KdV equation [18]. In addition to shallow water waves, the equation is applicable to the study of drift waves in plasma or the Rossby waves in rotating fluids. Under certain conditions, it also provides a model of onedimensional transmitted waves.

The main mathematical difference between $\mathrm{KdV}$ and BBM models can be most readily appreciated by comparing the dispersion relation for the respective linearized equations. It can be easily seen that these relations are comparable only for small wave numbers and they generate drastically different responses to short waves. This is one of the reasons why, whereas existence and regularity theory for the $\mathrm{KdV}$ equation is difficult, the theory of the BBM equation is comparatively simple [19], where the BBM equation does not take into account dissipation and is nonintegrable [20$22]$. The KdV equation describes long nonlinear waves of small amplitude on the surface of inviscid ideal fluid [22]. 
The $\mathrm{KdV}$ equation is integrable by the inverse scattering transform. Solitons exist due to the balance between the weak nonlinearity and dispersion of the KdV equation. Soliton is a localized wave that has an infinite support or a localized wave with exponential wings. The solutions of the BBM equation and the $\mathrm{KdV}$ equation have been of considerable concern. Zabusky and Kruskal investigated the interaction of solitary waves and the recurrence of initial states [23]. The term soliton is coined to reflect the particle like behavior of the solitary waves under interaction. The interaction of two solitons emphasized the reality of the preservation of shapes and speeds and of the steady pulse like character of solitons [24-27].

This paper is organized as follows: an introduction is in Section 1. In Section 2, the problem formulations to derive the nonlinear BBM and $\mathrm{KdV}$ equations are formulated. The extended direct algebraic and sech-tanh methods are analyzed in Section 3. In Section 4, the traveling solutions of the $\mathrm{BBM}$ and $\mathrm{KdV}$ equations are obtained.

\section{Problem Formulation}

In water wave equations, a two-dimensional inviscid, incompressible fluid with constant gravitational field is considered. The physical parameters are scaled into the definition of space, $(x, y)$, time $t$ and the gravitational acceleration $g$ is in the negative $y$ direction. Let $h_{0}$ be the undisturbed depth of the fluid and let $y=\eta(x, t)$ represent the free surface of the fluid. We also assume that the motion is irrotational and let $\phi(x, y, t)$ denote the velocity potential $(u=\nabla \phi)$. The divergence-free condition on the velocity field implies that the velocity potential $\phi$ satisfies the Laplaces equation $[28,29]$ :

$$
\frac{\partial^{2} \phi}{\partial x^{2}}+\frac{\partial^{2} \phi}{\partial y^{2}}=0, \quad \text { at }-h_{0}<y<\eta(x, t) .
$$

On a solid fixed boundary, the normal velocity of the fluid must vanish. For a horizontal flat bottom, we have

$$
\frac{\partial \phi}{\partial y}=0 \quad \text { at } y=-h_{0}
$$

The boundary conditions at the free surface $y=\eta(x, t)$ are given by

$$
\begin{gathered}
\frac{\partial \phi}{\partial y}-\frac{\partial \eta}{\partial t}-\frac{\partial \phi}{\partial x} \frac{\partial \eta}{\partial x}=0 \\
\frac{\partial \phi}{\partial t}+\frac{1}{2}\left(\left(\frac{\partial \phi}{\partial x}\right)^{2}+\left(\frac{\partial \phi}{\partial y}\right)^{2}\right)+g \eta=0
\end{gathered}
$$

Equation (3) is a kinematic boundary condition, while (4) represents the continuity of pressure at the free surface, as derived from Bernoullis equation. The Laplace equation (1) and the boundary conditions (2) on the bottom are already linear and are independent of $\eta$. Moreover, $\eta$ can be eliminated from the linear versions of (3) and (4). The first order equations for $\phi$ in the form

$$
\begin{gathered}
\frac{\partial^{2} \phi}{\partial x^{2}}+\frac{\partial^{2} \phi}{\partial y^{2}}=0, \quad \text { at }-h_{0}<y<0, \\
\frac{\partial \phi}{\partial y}=0, \quad \text { at } y=-h_{0}, \\
\frac{\partial^{2} \phi}{\partial t^{2}}+g \frac{\partial \phi}{\partial y}=0, \quad \text { at } y=0 .
\end{gathered}
$$

The progressive wave solution of the first order system is

$$
\phi(x, y, t)=\varphi(y) e^{i(k x-w t)} .
$$

Then, (5) has the solution

$$
\varphi(y)=A \cosh k\left(y+h_{0}\right)+B \sinh k\left(y+h_{0}\right),
$$

where $A$ and $B$ are arbitrary constants. The boundary condition (6) implies $B=0$, while the remaining condition (7) gives the dispersion relation

$$
w^{2}=g k \tanh k h_{0} .
$$

The dispersive effects can be combined with nonlinear effects to give

$$
u_{t}+\frac{3}{2} \frac{c_{0}}{h_{0}} u u_{x}+\int_{-\infty}^{\infty} K(x-\xi) u_{\xi}(\xi, t) d \xi=0,
$$

where $u(x, t)$ is the water wave velocity and $h_{0}$ is the depth of the fluid and $c_{0}=\sqrt{g h_{0}}$, with a kernel, $K(x)$, that is given by

$$
K(x)=\frac{1}{2 \pi} \int_{-\infty}^{\infty} c(k) e^{i k x} d x .
$$

From Taylor expansion, the partial deferential equation (11) reduces to the $\mathrm{KdV}$ equation:

$$
u_{t}+c_{0} u_{x}+\frac{3}{2} \frac{c_{0}}{h_{0}} u u_{x}+\frac{1}{6} c_{0} h_{0}^{2} u_{x x x}=0 .
$$

The BBM model was introduced in [18] as an alternative to the KdV equation. The main argument is that the phase velocity $\omega / k$ and the group velocity $d \omega / d k$ in the KdV model are not bounded from below. In contrast, the BBM equation has a phase velocity and a group velocity both of which are bounded for all $k$. They also approach zero as $k \rightarrow \infty$.

The derivation of the KdV equation in [18] uses a scaling of the variables $u, x$, and $t$, and a perturbation expansion argument in such a way that the dispersive and the nonlinear effects become small. The main argument that was used in [18] to derive the BBM equation is that, to the first order in $\epsilon$, the scaled $\mathrm{KdV}$ equation is equivalent to

$$
u_{t}+u_{x}+u u_{x}-a^{2} u_{x x x}-b^{2} u_{x x t}=0 .
$$

While the derivation presented in [18] is formally valid, it is important to note that the particular choice of the mixed derivative $-u_{x x t}$ as a replacement of $u_{x x x}$ may seem arbitrary from the point of view of asymptotic expansions. Indeed, any admissible combination of these two terms could be valid based on the zero-order correspondence between the derivatives in space and time $\left(u_{t}=-u_{x}\right)$. 


\section{An Analysis of the Methods}

The following is given nonlinear partial differential equations (BBM and $\mathrm{KdV}$ equations) with two variables $x$ and $t$ as

$$
F\left(u, u_{t}, u_{x}, u_{x x}, u_{x x x}\right)=0
$$

can be converted to ordinary deferential equations:

$$
F\left(u, u^{\prime}, u^{\prime \prime}, u^{\prime \prime \prime}\right)=0
$$

by using a wave variable $\xi=x-c t$. The equation is integrated as all terms contain derivatives where integration constants are considered zeros.

3.1. Extended Direct Algebraic Methods. We introduce an independent variable, where $u=\phi(\xi)$ is a solution of the following third-order ODE:

$$
\phi^{\prime 2}= \pm \alpha \phi^{2}(\xi) \pm \beta \phi^{4}(\xi)
$$

where $\alpha, \beta$ are constants. We expand the solution of (16) as the following series:

$$
u(\xi)=\sum_{k=0}^{m} a_{k} \phi^{k}+\sum_{k=1}^{m} b_{k} \phi^{-k}
$$

where $m$ is a positive integer, in most cases, that will be determined. The parameter $m$ is usually obtained by balancing the linear terms of highest order in the resulting equation with the highest order nonlinear terms. Substituting from (18) into the ODE (16) results in an algebraic system of equations in powers of $\phi$ that will lead to the determination of the parameters $a_{k},(k=0,1, \ldots, m)$ and $c$ by using Mathematica.

3.2. The Sech-Tanh Method. We suppose that $u(x, t)=u(\xi)$, where $\xi=x-c t$ and $u(\xi)$ has the following formal travelling wave solution:

$$
u(\xi)=\sum_{i=1}^{n} \operatorname{sech}^{i-1} \xi\left(A_{i} \operatorname{sech} \xi+B_{i} \tanh \xi\right)
$$

where $A_{0}, A_{1}, \ldots, A_{n}$ and $B_{1}, \ldots, B_{n}$ are constants to be determined.

Step 1. Equating the highest-order nonlinear term and highest-order linear partial derivative in (16) yields the value of $n$.

Step 2. Setting the coefficients of $\left(\operatorname{sech}^{j} \tanh ^{i}\right)$ for $i=0,1$ and $j=1,2, \ldots$ to zero, we have the following set of overdetermined equations in the unknowns $A_{0}, A_{i}, B_{i}$, and $c$ for $i=1,2, \ldots, n$.

Step 3. Using Mathematica and Wu s̀ elimination methods, the algebraic equations in Step 2 can be solved.

\section{Application of the Methods}

4.1. Exact Solutions for KdV Equation. We will employ the proposed methods to solve the KdV equation:

$$
u_{t}+c_{0} u_{x}+\frac{3}{2} \frac{c_{0}}{h_{0}} u u_{x}+\frac{1}{6} c_{0} h_{0}^{2} u_{x x x}=0 .
$$

By assuming travelling wave solutions of the form $u(x, t)=$ $u(\xi), \xi=x-c t,(20)$ is equivalent to

$$
\left(c_{0}-c\right) u^{\prime}+\frac{3}{2} \frac{c_{0}}{h_{0}} u u^{\prime}+\frac{1}{6} c_{0} h_{0}^{2} u^{\prime \prime \prime}=0 .
$$

Balancing $u^{\prime \prime \prime}$ with $u u^{\prime}$ in (21) gives $m=2$; then

$$
u(\xi)=a_{0}+a_{1} \phi+a_{2} \phi^{2}+\frac{b_{1}}{\phi}+\frac{b_{2}}{\phi^{2}} .
$$

Substituting into (21) and collecting the coefficient of $\phi$, we obtain a system of algebraic equations for $a_{0}, a_{1}, a_{2}, b_{1}, b_{2}$, and $c$. Solving this system gives the following real exact solutions.

Case I. Suppose that

$$
\phi^{\prime 2}=-\alpha \phi^{2}(\xi)+\beta \phi^{4}(\xi)
$$

by comparing them with the coefficients of $\phi^{i}(i=$ $-5,-4,-3,-2,-1,0,1,2,3)$ where $\alpha>0$ :

$$
\phi(\xi)=\sqrt{\frac{\alpha}{\beta}} \sec \left(\sqrt{\alpha} \xi+\xi_{0}\right),
$$

where $\xi_{0}$ is constant of integration; then we have

$$
\begin{aligned}
& a_{0}=\frac{2}{9 c_{0}}\left(3 c h_{0}-3 c_{0} h_{0}+2 \alpha c_{0} h_{0}^{3}\right), \\
& a_{2}=-\frac{4}{3} \beta h_{0}^{3}, \quad a_{1}=b_{1}=b_{2}=0 .
\end{aligned}
$$

In this case, the generalized soliton solution can be written as

$$
\begin{aligned}
u_{1}(x, t)= & \frac{2}{9} h_{0} \\
& \times\left(-3+3 \frac{c}{c_{0}}+2 \alpha h_{0}^{2}\left(1-3 \sec ^{2}\left(\sqrt{\alpha}(x-c t)+\xi_{0}\right)\right)\right) .
\end{aligned}
$$

Figure 1 shows the travelling wave solutions with ( $\alpha=$ $\left.0.16, \beta=0.25, k=0.5, h_{0}=0.25, c_{0}=2\right)$ in the interval $[-10,10]$ and time in the interval $[0,0.1]$.

Case II. Suppose that

$$
\phi^{\prime 2}=\alpha \phi^{2}(\xi)-\beta \phi^{4}(\xi),
$$

by comparing them with the coefficients of $\phi^{i}$ and $\alpha>0$ under condition $\phi(0)=\sqrt{\alpha / \beta}$, so

$$
\phi(\xi)=\sqrt{\frac{\alpha}{\beta}} \operatorname{sech}(\sqrt{\alpha} \xi) ;
$$




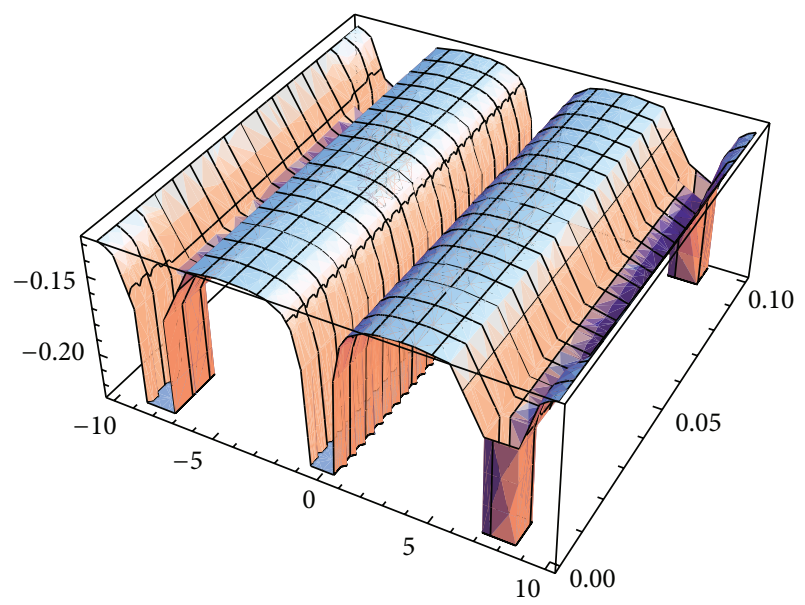

Figure 1: Travelling waves solutions (26) are plotted: periodic solitary waves.

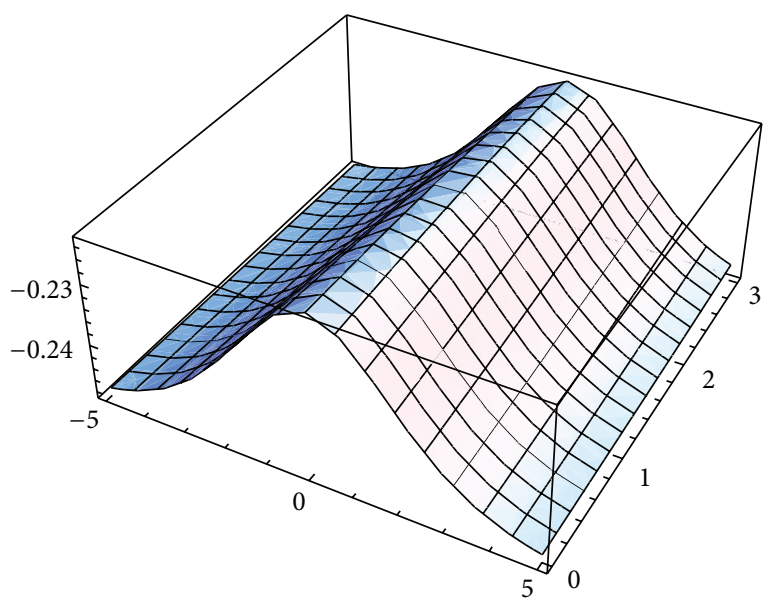

FIGURE 2: Travelling waves solutions (30) are plotted: bright solitary waves.

then we have

$$
\begin{aligned}
& a_{0}=\frac{2}{9 c_{0}}\left(-3 c_{0} h_{0}+3 c h_{0}-2 \alpha c_{0} h_{0}^{3}\right), \\
& a_{2}=-\frac{4}{3} \beta h_{0}^{3}, \quad a_{1}=b_{1}=b_{2}=0 .
\end{aligned}
$$

In this case, the generalized soliton solution can be written as

$$
u_{2}(x, t)=\frac{2}{9} h_{0}\left(-3+3 \frac{c}{c_{0}}-2 \alpha h_{0}^{2}\left(1+3 \operatorname{sech}^{2}(\sqrt{\alpha}(x-c t))\right)\right) \text {. }
$$

Figure 2 shows the travelling wave solutions with $(\alpha=$ $\left.0.16, k=0.25, h_{0}=0.5, c_{0}=0.9\right)$ in the interval $[-5,5]$ and time in the interval $[0,3]$. if

The stability of soliton solution is stable at $t=0, \alpha=0.16$

$$
k>c_{0}\left[1-0.048 h_{0}^{2}\right]
$$

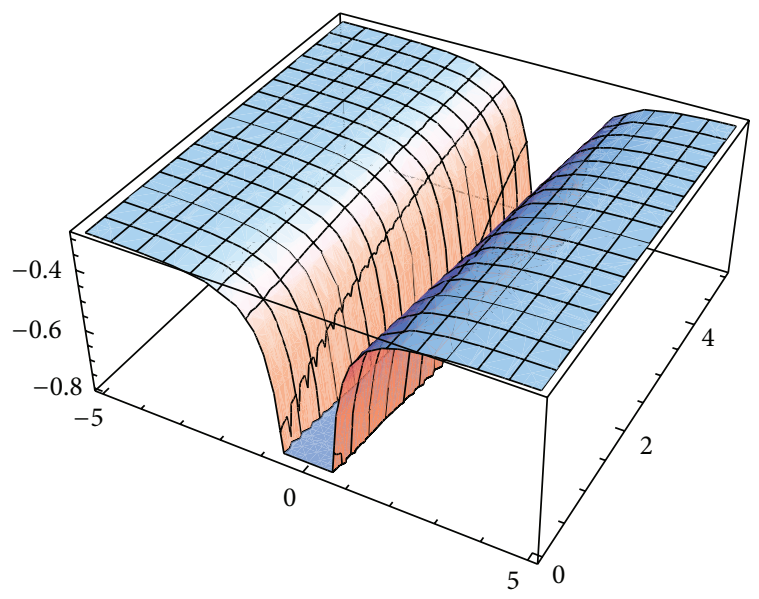

FIGURE 3: Travelling waves solutions (35) are plotted: dark solitary waves.

Case III. Suppose that

$$
\phi^{\prime 2}=\alpha \phi^{2}(\xi)+\beta \phi^{4}(\xi),
$$

by comparing them with the coefficients of $\phi^{i}$ and $\alpha>0$ under condition $\beta=1 / 4$ so

$$
\phi(\xi)=2 \sqrt{\alpha} \operatorname{csch}(\sqrt{\alpha} \xi)
$$

and we have

$$
\begin{aligned}
& a_{0}=\frac{2}{9 c_{0}}\left(-3 c_{0} h_{0}+3 c h_{0}-2 \alpha c_{0} h_{0}^{3}\right), \\
& a_{2}=-\frac{h_{0}^{3}}{3}, \quad a_{1}=b_{1}=b_{2}=0 .
\end{aligned}
$$

In this case, the generalized soliton solution can be written as

$$
u_{3}(x, t)=\frac{2}{9} h_{0}\left(-3+3 \frac{c}{c_{0}}-2 \alpha h_{0}^{2}\left(1+3 \operatorname{csch}^{2}[\sqrt{\alpha}(x-c t)]\right)\right) \text {. }
$$

Figure 3 shows the travelling wave solutions with $(\alpha=$ $\left.0.25, k=0.16, h_{0}=0.5, c_{0}=0.9\right)$ in the interval $[-5,5]$ and time in the interval $[0,5]$.

\section{Using Sech-Tanh Method. Consider}

$$
\begin{aligned}
u(\xi)= & A_{0}+A_{1} \operatorname{sech} \xi+B_{1} \tanh \xi+A_{2} \operatorname{sech} \xi^{2} \\
& +B_{2} \tanh \xi \operatorname{sech} \xi
\end{aligned}
$$

Substituting from (36) into (21), setting the coefficients of $\left(\operatorname{sech}^{j} \tanh ^{i}\right.$ ) for $i=0,1$ and $j=1,2,3,4$ to zero, we have the following set of overdetermined equations in the unknowns $A_{0}, A_{1}, A_{2}, B_{1}, B_{2}$, and $c$. Solve the set of equations 


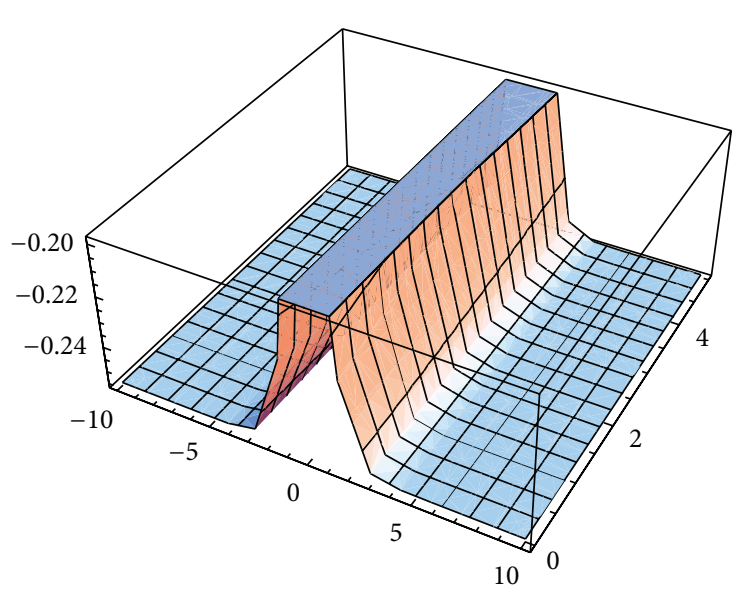

(a)

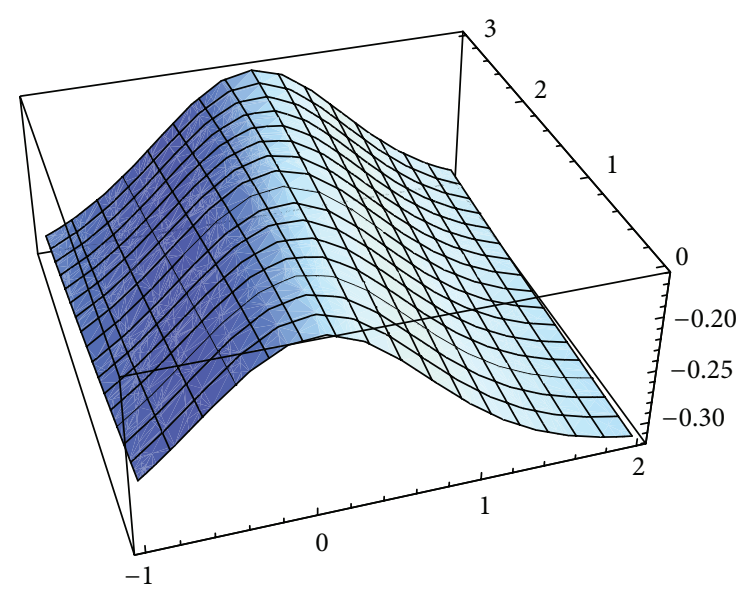

(b)

FIGURE 4: Travelling waves solutions (40) are plotted: bright solitary waves.

of coefficients of $\left(\operatorname{sech}^{j} \tanh ^{i}\right)$, by using Mathematica and Wu s̀ elimination method; we obtain the following solutions:

$$
\begin{gathered}
\text { (i) } A_{0}=-\frac{h_{0}\left(-6 k+6 c_{0}+c_{0} h_{0}^{2}\right)}{9 c_{0}} \\
A_{2}=\frac{2 h_{0}^{3}}{3}, \quad B_{2}=\mp \frac{2}{3} i h_{0}^{3}, \quad A_{1}=B_{1}=0,
\end{gathered}
$$

and then the solution of $\mathrm{KdV}$ equation as

$$
\begin{aligned}
& u_{4}(x, t)=\frac{h_{0}}{9 c_{0}}\left(6 k+c_{0}\right. \\
& \times\left(-6+h_{0}^{2}(-1+6 \operatorname{sech}[x-k t]\right. \\
& \times(\operatorname{sech}[x-k t] \\
& \pm i \tanh [x-k t]))) .
\end{aligned}
$$

This soliton solution is stable if

$$
\text { (ii) } \begin{aligned}
A_{0} & =\frac{2}{9}\left(-3 h_{0}+\frac{3 k h_{0}}{c_{0}}-2 h_{0}^{3}\right), \\
A_{2} & =\frac{4 h_{0}^{3}}{3}, \quad A_{1}=B_{1}=B_{2}=0,
\end{aligned}
$$

and the solution of $\mathrm{KdV}$ equation is

$$
u_{5}(x, t)=\frac{2}{9} h_{0}\left(-3+\frac{3 k}{c_{0}}+\left(-2+6 \operatorname{sech}^{2}[x-k t]\right) h_{0}^{2}\right) .
$$

Figure 4(a) shows the travelling wave solutions with $(k=$ $\left.0.1, h_{0}=0.5, c_{0}=0.25\right)$ in the interval $[-10,10]$ and time in the interval $[0,5]$.
Figure 4(b) shows the travelling wave solutions with $(k=$ $\left.0.16, h_{0}=0.5, c_{0}=0.9\right)$ in the interval $[-1,2]$ and time in the interval $[0,3]$.

This soliton solution is stable if

$$
k>c_{0}\left(1+\frac{\left(13+7 e^{20}\right) h_{0}^{2}}{15\left(1+e^{20}\right)}\right) .
$$

4.2. Solutions for Benjamin-Bona-Mahony Equation. The Benjamin-Bona-Mahony equation (14) can be transformed to ODE as

$$
(k-c) u^{\prime}+k u u^{\prime}+\left(b^{2} k^{2} c-a^{2} k^{3}\right) u^{\prime \prime \prime}=0 .
$$

Balancing $u^{\prime \prime \prime}$ with $u u^{\prime}$ in (21) gives $m=2$.

First Case. Let finite expansion

$$
u(\xi)=A_{0}+A_{1} \operatorname{sech} \xi+A_{2} \operatorname{sech}^{2} \xi+B_{1} \cosh \xi+B_{2} \cosh ^{2} \xi .
$$

Substituting from (43) into (42) and setting the coefficients of $\left(\operatorname{sech}^{j} \cosh ^{i}\right)$ for $i, j=1,2,3,4$ to zero, we have the following set of overdetermined equations in the unknowns $A_{0}, A_{1}$, $B_{1}$, and $B_{2}$. By solving the set of equations of coefficients of $\left(\operatorname{sech}^{j} \cosh ^{i}\right)$ by using Mathematica method, we obtain the following solution:

$$
\begin{gathered}
A_{1}=B_{1}=B_{2}=0, \quad A_{0}=\frac{c-k-4 b^{2} k^{2} c+4 a^{2} k^{3}}{k}, \\
A_{2}=12\left(b^{2} c k-a^{2} k^{2}\right) .
\end{gathered}
$$

The exact soliton solution of Benjamin-Bona-Mahony equation is

$$
\begin{aligned}
u(x, t)= & \left(\frac{c-k-4 b^{2} k^{2} c+4 a^{2} k^{3}}{k}\right) \\
& +12\left(b^{2} c k-a^{2} k^{2}\right) \operatorname{sech}^{2}(k x-c t) .
\end{aligned}
$$




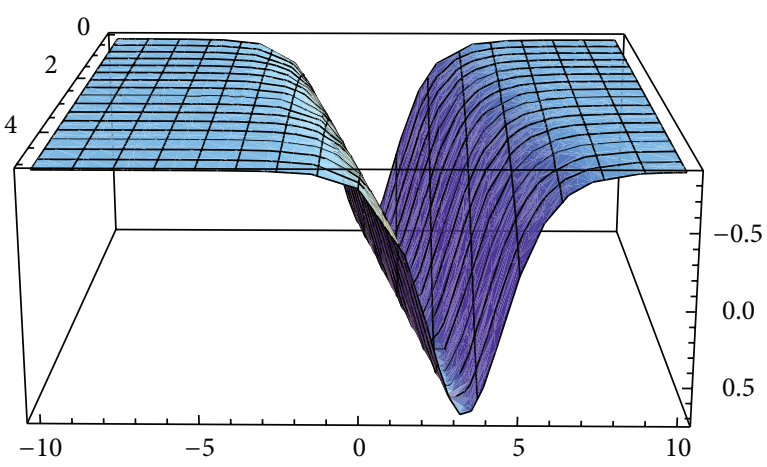

FIGURE 5: Travelling waves solutions (45) are plotted: dark solitary waves.

Figure 5 shows the travelling wave solutions with $(k=$ $\left.0.6, c=0.4, a^{2}=9 / 10, b^{2}=19 / 10\right)$ in the interval $[-10,10]$ and time in the interval $[0,5]$.

Second Case. Let finite expansion

$$
u(\xi)=A_{0}+A_{1} \operatorname{coth} \xi+A_{2} \operatorname{coth}^{2} \xi+B_{1} \tanh \xi+B_{2} \tanh ^{2} \xi .
$$

Then, we obtain the following solutions:

$$
\begin{gathered}
A_{1}=B_{1}=0, \quad A_{0}=\frac{c-k+8 b^{2} k^{2} c-8 a^{2} k^{3}}{k}, \\
A_{2}=B_{2}=-12\left(b^{2} c k-a^{2} k^{2}\right)
\end{gathered}
$$

so that the exact soliton solution be

$$
\begin{aligned}
u(x, t)= & \left(\frac{c-k+8 b^{2} k^{2} c-8 a^{2} k^{3}}{k}\right)-12\left(b^{2} c k-a^{2} k^{2}\right) \\
& \times\left(\tanh ^{2}(k x-c t)+\operatorname{coth}^{2}(k x-c t)\right) .
\end{aligned}
$$

Figure 6 shows the travelling wave solutions with $(k=$ $\left.0.6, c=0.4, a^{2}=9 / 10, b^{2}=19 / 10\right)$ in the interval $[-10,10]$ and time in the interval $[0,5]$.

\section{Conclusion}

By implementing the extended direct algebraic and modified sech-tanh methods, we presented new traveling wave solutions of the KdV and BBM equations. We obtained the water wave velocity potential of $\mathrm{KdV}$ equation in periodic form and bright and dark solitary wave solutions by using the extended direct algebraic method. Using the modified sechtanh method, the water wave velocity of KdV equation in form of bright and dark solitary wave solutions. The water wave velocity potentials of BBM equation are deduced in form of dark solitary wave solutions. The structures of the obtained solutions are distinct and stable.

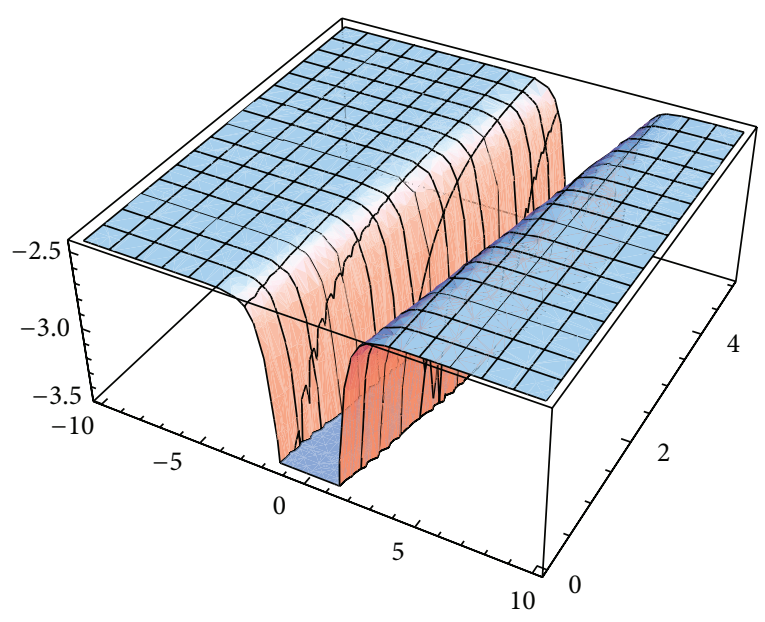

FIgURE 6: Travelling waves solutions (48) are plotted: dark solitary waves.

\section{Conflict of Interests}

The authors declare that there is no conflict of interests regarding the publication of this paper.

\section{References}

[1] A. R. Seadawy, "New exact solutions for the KdV equation with higher order nonlinearity by using the variational method," Computers \& Mathematics with Applications, vol. 62, no. 10, pp. 3741-3755, 2011.

[2] M. A. Helal and A. R. Seadawy, "Benjamin-Feir instability in nonlinear dispersive waves," Computers \& Mathematics with Applications, vol. 64, no. 11, pp. 3557-3568, 2012.

[3] A. R. Seadawy and K. El-Rashidy, "Traveling wave solutions for some coupled nonlinear evolution equations," Mathematical and Computer Modelling, vol. 57, no. 5-6, pp. 1371-1379, 2013.

[4] F. Yan, H. Liu, and Z. Liu, "The bifurcation and exact travelling wave solutions for the modified Benjamin-BONa-Mahoney (mBBM) equation," Communications in Nonlinear Science and Numerical Simulation, vol. 17, no. 7, pp. 2824-2832, 2012.

[5] M. J. Ablowitz and P. A. Clarkson, Soliton, Nonlinear Evolution Equations and Inverse Scattering, Cambridge University Press, New York, NY, USA, 1991.

[6] R. Hirota, "Exact solution of the korteweg-de vries equation for multiple Collisions of solitons," Physical Review Letters, vol. 27, no. 18, pp. 1192-1194, 1971.

[7] A.-M. Wazwaz, "New travelling wave solutions of different physical structures to generalized BBM equation," Physics Letters A: General, Atomic and Solid State Physics, vol. 355, no. 4-5, pp. 358-362, 2006.

[8] M. R. Miurs, Backlund Transformation, Springer, Berlin, Germany, 1978.

[9] X. Liu, L. Tian, and Y. Wu, "Exact solutions of the generalized Benjamin-BONa-Mahony equation," Mathematical Problems in Engineering, vol. 2010, Article ID 796398, 5 pages, 2010.

[10] Ş. Kuru, "Traveling wave solutions of the BBM-like equations," Journal of Physics A: Mathematical and Theoretical, vol. 42, no. 37, Article ID 375203, 12 pages, 2009.

[11] P. G. Estévez, Ş. Kuru, J. Negro, and L. M. Nieto, “Travelling wave solutions of the generalized Benjamin-Bona-Mahony 
equation," Chaos, Solitons \& Fractals, vol. 40, no. 4, pp. 20312040, 2009.

[12] A. Wazwaz, "Exact solutions with compact and noncompact structures for the one-dimensional generalized BenjaminBona-Mahony equation," Communications in Nonlinear Science and Numerical Simulation, vol. 10, no. 8, pp. 855-867, 2005.

[13] Q.-H. Feng, F.-W. Meng, and Y.-M. Zhang, "Traveling wave solutions for two nonlinear evolution equations with nonlinear terms of any order," Chinese Physics B, vol. 20, no. 12, Article ID 120202, 2011.

[14] X. Liu, L. Tian, and Y. Wu, "Exact solutions of four generalized Benjamin-Bona-Mahony equations with any order," Applied Mathematics and Computation, vol. 218, no. 17, pp. 8602-8613, 2012.

[15] S. Abbasbandy and A. Shirzadi, "The first integral method for modified Benjamin-Bona-Mahony equation," Communications in Nonlinear Science and Numerical Simulation, vol. 15, no. 7, pp. 1759-1764, 2010.

[16] B. Hong and D. Lu, "New exact solutions for the generalized BBM and Burgers-BBM equations," World Journal of Modelling and Simulation, vol. 4, pp. 243-249, 2008.

[17] W. Hereman, "Shallow water waves and solitary waves," in Mathematics of Complexity and Dynamical Systems, pp. 15201532, 2011.

[18] J. Nickel, "Elliptic solutions to a generalized BBM equation," Physics Letters. A, vol. 364, no. 3-4, pp. 221-226, 2007.

[19] K. Singh, R. K. Gupta, and S. Kumar, "Benjamin-Bona-Mahony (BBM) equation with variable coefficients: similarity reductions and Painlevé analysis," Applied Mathematics and Computation, vol. 217, no. 16, pp. 7021-7027, 2011.

[20] T. B. Benjamin, J. L. Bona, and J. J. Mahony, "Model equations for long waves in nonlinear dispersive systems," Philosophical Transactions of the Royal Society of A: Mathematical and Physical Sciences, vol. 272, no. 1220, pp. 47-78, 1972.

[21] J. Saut and N. Tzvetkov, "Global well-posedness for the KP-BBM equations," Applied Mathematics Research eXpress, no. 1, pp. 116, 2004.

[22] V. Varlamov and Y. Liu, "Cauchy problem for the Ostrovsky equation," Discrete and Continuous Dynamical Systems A, vol. 10, no. 3, pp. 731-753, 2004.

[23] N. J. Zabusky and M. D. Kruskal, "Interaction of "solitons" in a collisionless plasma and the recurrence of initial states," Physical Review Letters, vol. 15, no. 6, pp. 240-243, 1965.

[24] A. Wazwaz and M. A. Helal, "Nonlinear variants of the BBM equation with compact and noncompact physical structures," Chaos, Solitons and Fractals, vol. 26, no. 3, pp. 767-776, 2005.

[25] M. A. Helal and A. R. Seadawy, "Variational method for the derivative nonlinear Schrödinger equation with computational applications," Physica Scripta, vol. 80, no. 3, Article ID 035004, 2009.

[26] A. R. Seadawy, "Exact solutions of a two-dimensional nonlinear Schrödinger equation," Applied Mathematics Letters, vol. 25, no. 4, pp. 687-691, 2012.

[27] A. R. Seadawy, "Stability analysis for Zakharov-Kuznetsov equation of weakly nonlinear ion-acoustic waves in a plasma," Computers \& Mathematics with Applications, vol. 67, no. 1, pp. 172-180, 2014.

[28] A. Jeffrey and T. Kakutani, "Weak nonlinear dispersive waves: a discussion centered around the Korteweg-de Vries equation," SIAM Review, vol. 14, pp. 582-643, 1972.
[29] R. Fetecau and D. Levy, "Approximate model equations for water waves," Communications in Mathematical Sciences, vol. 3, no. 2, pp. 159-170, 2005. 


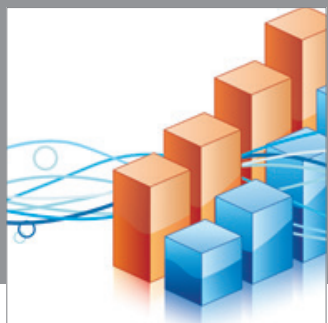

Advances in

Operations Research

mansans

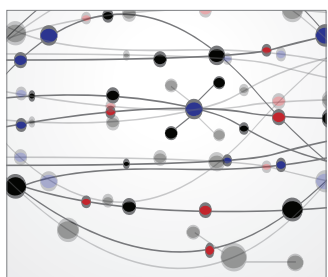

The Scientific World Journal
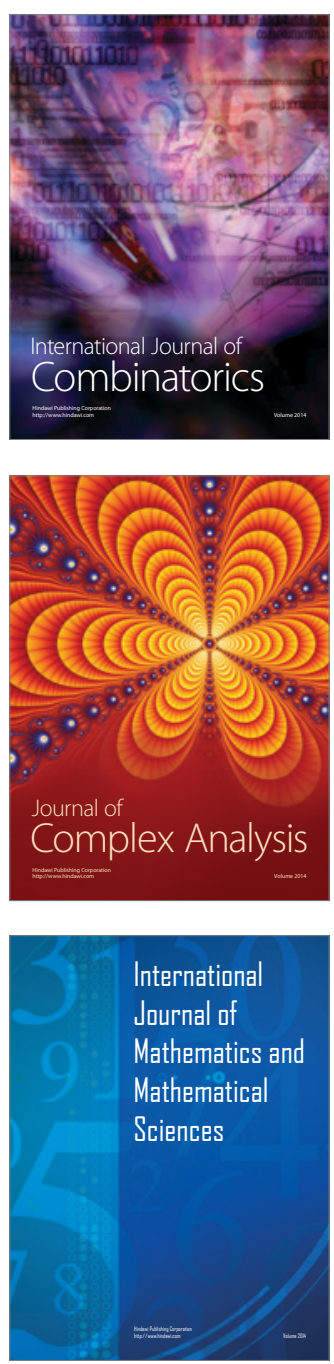
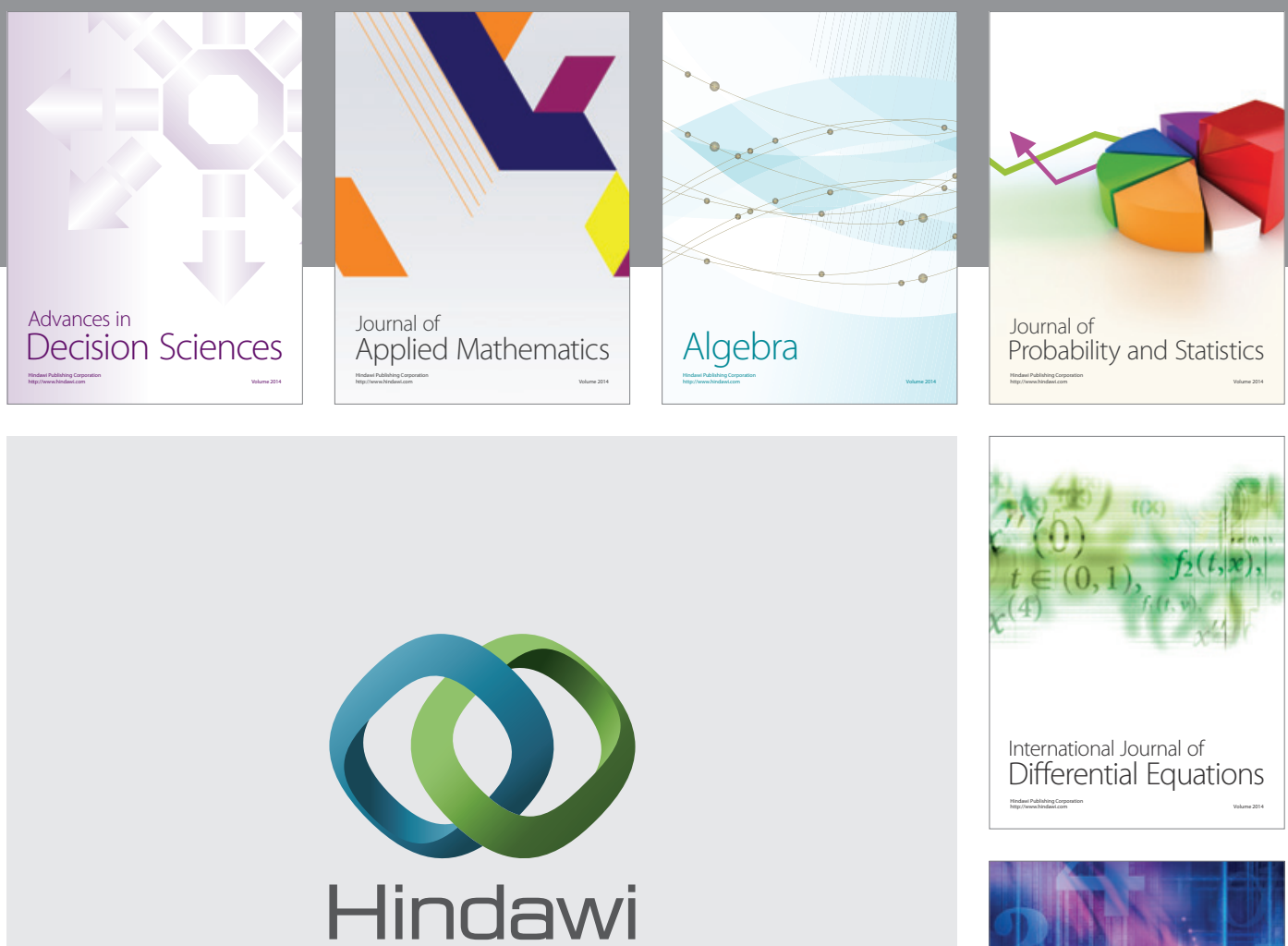

Submit your manuscripts at http://www.hindawi.com
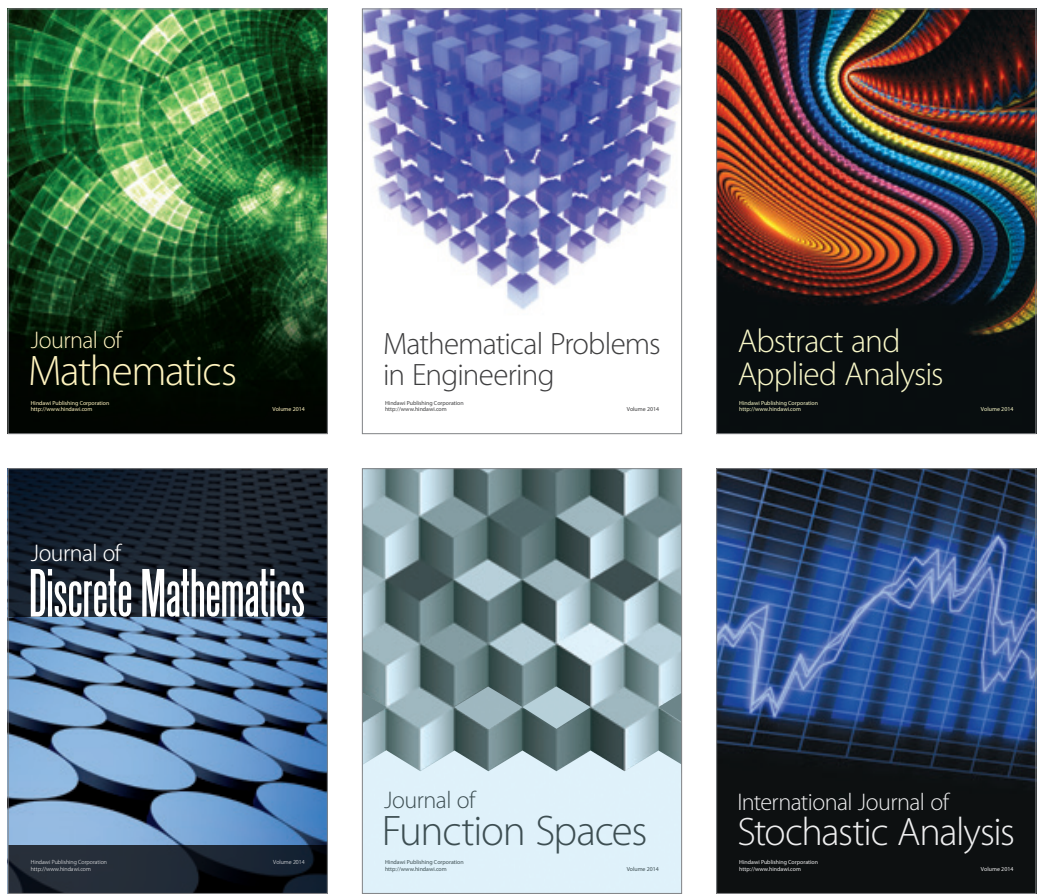

Journal of

Function Spaces

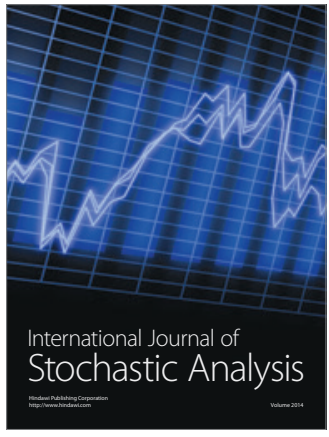

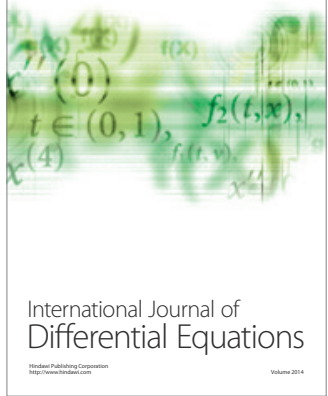
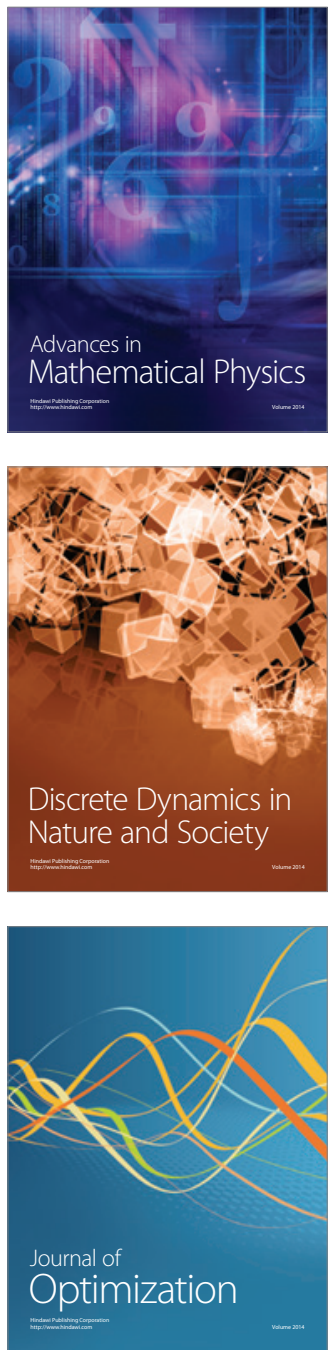